\title{
Study of Occurrence Characteristics of Antibiotic Resistance Genes in Pig manure during Anaerobic Digestion and Digestate Fertilization
}

\author{
PAN Xin-rong, CHEN Lei, YU Heng, and ZUO Jian-e* \\ State Key Joint Laboratory of Environment Simulation and Pollution Control, School of Environment, Tsinghua University, Beijing, \\ 100084, China
}

\begin{abstract}
Antibiotic resistance genes (ARGs) existing in livestock and poultry manure have the risk to spread and proliferate. This might endanger people's health. The common treatment of livestock and poultry manure is anaerobic digestion. But the change of ARGs during anaerobic digestion require further study, and the effect of digestate fertilization to the antibiotic resistance of cropland soil is still unclear. This study investigated the pig manure, biogas liquid, biogas residue, and cropland soils fertilized with and without digestate. The results showed that, the relative abundance of ARGs in biogas residue was much higher than other samples. The average relative abundance was $1.46 \times 10^{-1}$ copy ratio (copy of ARG/copy of $16 \mathrm{~S}$ rRNA gene), and the total relative abundance was 3.07 copy ratio. There were $21 \mathrm{ARGs}$ detected in the 5 samples. 11 of them were shared by the 5 samples. The main ARGs were aminoglycoside, chloramphenicol, sulfonamide, tetracycline, and multidrug. Aminoglycoside had the highest relative abundance, and the total relative abundance in all samples was 1.18 copy ratio. Anaerobic digestion increased the total relative abundance of ARGs in pig manure from $1.14 \times 10^{-1}$ to $1.70 \times 10^{-1}$ copy ratio. Fertilization of digestate increased the total relative abundance of AGRs in soil from $3.27 \times 10^{-1}$ to $7.29 \times 10^{-1}$ copy ratio.
\end{abstract}

\section{Introduction}

Due to the heavy and widely use of antibiotics in the process of livestock and poultry breeding, livestock manure has become an important enrichment reservoir and source of transmission for ARGs and antibiotic resistance bacteria (ARB) [1]. The content of organic matter in livestock manure is high. Microorganisms are easy to breed and reproduce, and at the same time, heavy metals and antibiotics may remain, which will greatly promote the formation and spread of ARGs [2]. Studies have detected chloramphenicol, sulfonamides, erythromycin, and tetracycline ARGs occurring in livestock and poultry manure [3, 4]. There are also articles that detected the occurrence of ARGs in soil samples directly applied with livestock and poultry manure, which also confirmed the presence of resistant bacteria in vegetables, which greatly increases our exposure to ARGs risk [5]. Therefore, if improperly handled, ARGs and ARB in livestock and poultry manure will spread to the environment. In the process of spreading, drug-resistant pathogens and even super bacteria may be formed, harming human health $[6,7]$.

Anaerobic digestion is a common treatment method for livestock and poultry manure. It has the advantages of low energy consumption, high reactor load, the ability to generate methane as an energy substance, and digestate as a soil amendment [8]. Experiments show that the anaerobic digestion process can remove ARGs and $\mathrm{ARB}$ in organic solid waste, and the temperature and solid retention time are the key factors affecting removal efficiency [9, 10]. By adding $\mathrm{FeCl}_{2}$, not only the production of methane can be increased, but also the removal rate of AGRs can be improved [11]. However, some studies have shown that ARGs did not decrease during anaerobic digestion, and some ARGs even increased significantly $[12,13]$. For example, in the anaerobic digestion process of pig manure, the addition of magnetite increased the methane production, but the tetracycline ARG will be enriched in the digestate [14]. On the other hand, the use of livestock and poultry manure treatment products as farmland fertilizers may also increase the antibiotic resistance of soil, although the relative abundance of ARGs in the idle period declined. The ARGs in farmland soils applied with digestate throughout the year will remain stable [15]. At present, most research focus on a few ARGs, while a more detailed and in-depth analysis and comparison of ARGs can be conducted through metagenomics. Especially in the actual treatment process of livestock and poultry manure, whether ARGs contained in livestock manure can be effectively removed during the anaerobic digestion process, and whether the digestate should be used in the process of farmland utilization is still opaque. The spread of drug-resistant pollution still requires further investigation and analysis.

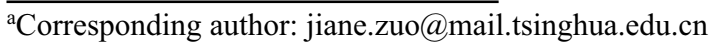


China is a big country in livestock and poultry breeding. According to estimation, in 2015, China's livestock and poultry manure (including urine) reached 6.554 billion tons, of which pig manure was 3.561 billion tons, accounting for $54 \%$ of livestock and poultry manure. In this study, pig manure was researched to investigate the occurrence and change of ARGs in pig manure during anaerobic digestion and farmland utilization. By comparing the difference of ARGs in soil with anaerobic digestion liquid and conventional fertilizer, the potential risks of anaerobic digestion in the farmland application was evaluated, and scientific basis for the treatment and disposal of livestock manure will be provided.

\section{METHODS AND MATERIALS}

\subsection{Sample collection method}

Taking the anaerobic digestion of pig manure as the research object, the digestion reactor treat $775 \mathrm{~m}^{3}$ of pig manure and urine every day. The size of the reactor: diameter $\times$ height $=13.5 \mathrm{~m} \times 10 \mathrm{~m}$. The operating temperature of the digestion tank is $35{ }^{\circ} \mathrm{C}$. Anaerobic biogas liquid and the biogas residue after fermentation is used to irrigate the wheat fields. The amount of biogas liquid produced per day is about $615 \mathrm{~m}^{3}$, and the amount of biogas residue is about $10 \mathrm{t}$. The pig manure and anaerobic digestion biogas liquid and residue were collected separately. The biogas liquid and biogas residue were obtained by sedimentation and separation for detection and analysis. At the same time, the wheat field soil with and without application anaerobic digestate were collected for analysis. Pig manure and anaerobic digestate gathered $500 \mathrm{~mL}$ sample at 3 times of a day seperatively, then mixed as the test sample. The soil samples were mixed with $200 \mathrm{~g}$ soil around and in the middle of the farmland. Liquid samples were stored in polyethylene bottles, and soil samples were stored in sealed bags. All the samples were sent to the laboratory immediately. The samples used for testing microorganisms were stored in $-80{ }^{\circ} \mathrm{C}$ refrigerator, and the samples used for testing other indicators were stored in $4{ }^{\circ} \mathrm{C}$ refrigerator.

Table 1. The corresponding of samples and nomenclature

\begin{tabular}{cc}
\hline Sample name & Sample type \\
\hline Pin & Pig manure \\
Pup & Biogas liquid \\
Pdown & Biogas residue \\
Psn & Soil without digestate from pig \\
manure \\
Psf & Soil with digestate from pig manure \\
\hline
\end{tabular}

\subsection{Analysis of conventional indicators}

The $\mathrm{pH}$, conductivity, ammonia nitrogen $\left(\mathrm{NH}_{4}^{-}-\mathrm{N}\right)$, total nitrogen (TN), total phosphorus (TP), TS (total solids), SCOD (soluble chemical organic demand), TCOD (total chemical organic demand) of the pig manure, biogas liquid, and biogas residue were analyzed. The $\mathrm{pH}$ was tested with a Mettler F2 pH meter, and the conductivity was tested with a Raymagnet DDS-307A conductivity meter. Other indicators were processed in accordance with the national standard method, and the absorbance was tested with an ultraviolet-visible spectrophotometer (Hach, DR6000). The pH, TN, TP, organic matter content, total salt content and conductivity in the soil were commissioned to the college platform.

Table 2. Basic characteristics of liquid samples

\begin{tabular}{ccccccc}
\hline Samples & $\begin{array}{c}\text { TCOD } \\
(\mathrm{mg} / \mathrm{L})\end{array}$ & $\begin{array}{c}\text { SCOD } \\
(\mathrm{mg} / \mathrm{L})\end{array}$ & $\mathrm{pH}$ & Conductivity $(\mathrm{ms} / \mathrm{cm})$ & $\begin{array}{c}\mathrm{NH}_{4}^{-}- \\
\mathrm{N} \\
(\mathrm{mg} / \mathrm{L})\end{array}$ & $\begin{array}{c}\mathrm{TP} \\
(\mathrm{mg} / \mathrm{L})\end{array}$ \\
\hline Pin & 6000 & 2000 & 7.09 & 6.22 & 276 & 320 \\
Pup & 5400 & 2600 & 7.29 & 5.59 & 309 & 330 \\
Pdown & 96800 & 2000 & 7.47 & 10.25 & 926 & 320 \\
\hline
\end{tabular}

Table 3. Basic characteristics of soil samples

\begin{tabular}{cccccccc}
\hline Samples & $\mathrm{pH}$ & $\begin{array}{c}\text { Soil } \\
\text { potassium } \\
(\mathrm{g} / \mathrm{kg})\end{array}$ & $\begin{array}{c}\text { Salinity } \\
(\mathrm{g} / \mathrm{kg})\end{array}$ & $\begin{array}{c}\text { Organic } \\
\text { matter } \\
(\mathrm{g} / \mathrm{kg})\end{array}$ & $\begin{array}{c}\mathrm{TP} \\
(\mathrm{g} / \mathrm{kg})\end{array}$ & $\begin{array}{c}\mathrm{TN} \\
(\mathrm{g} / \mathrm{kg})\end{array}$ & $\begin{array}{c}\text { Moisture } \\
(\%)\end{array}$ \\
\hline Psn & 6.96 & 0.27 & 0.97 & 18.90 & 0.69 & 1.58 & 6.65 \\
Psf & 6.92 & 0.33 & 3.88 & 13.70 & 0.80 & 1.61 & 6.01 \\
\hline
\end{tabular}

\subsection{DNA extraction and sequencing}

All samples were commissioned to Novogene Technology Co., Ltd. (Tianjin, China) for DNA extraction. The sample DNA was extracted by CTAB method, and then analyzed by agarose gel electrophoresis (AGE) for DNA purity and integrity. The qualified DNA samples performed gene library preparation, then used Qubit 2.0 fluorescence spectrophotometer (Life Technologies, Germany) for preliminary quantification, diluted the library to $2 \mathrm{ng} / \mathrm{ul}$, and used Agilent 2100 Bioanalyzer (Agilent Technologies, USA) to detect the inserted fragments of the library. After the library was qualified, the different libraries were sequenced according to the effective concentration and the target off-machine data volume. All DNA samples were sequenced on the Illumina MiSeq platform (PE250 strategy) of Novogene using 
$150 \mathrm{bp}$ paired-end sequencing. Sequencing a sample will obtain about $6 \mathrm{G}$ data. The sequencing data was evaluated, and unqualified original sequences were eliminated, and then subsequent analysis was performed.

\subsection{Metagenomic sequencing and analysis}

Based on the metagenomics method of secondgeneration high-throughput sequencing, the SARG 2.2 database (Structured ARG reference database 2.2) was used to annotate the ARGs sequence. First, the potential ARGs sequence was pre-screened from the short sequence of metagenomic data by the UBLAST tool, and then uploaded the potential ARGs sequences to the ARGs standardized online analysis platform (ARGsOAP) for analysis and comparison. Then, used BLASTX to annotate and classify the pre-screened potential ARG sequences, set the ending e value to $10^{-7}$ and the sequence similarity to $80 \%$. The sequence length exceeds 38 amino acids. The relative abundance of ARG types/subtypes was expressed by the ratio of ARG copy number to $16 \mathrm{~S}$ rRNA gene copy number.

Using excel 2013, origin 2018 software, and Majorbio cloud platform to organize, analyze and graph the data.

\section{RESULTS AND DISCUSSION}

\subsection{Comparison of the relative abundance of total ARGs in different samples}

As shown in Figure 1, a total of 21 ARGs were detected in all samples, 11 of which were present in all samples. At the same time, 12 ARGs coexisted in pig manure feed and biogas liquid and residue, and 15 ARGs were found both in soil samples with and without application of anaerobic digestate.

As shown in Figure 2, the distribution range of ARGs in the samples was relatively wide. Among the 5 samples, the total relative abundance of ARGs in anaerobic digestion biogas residue was the highest, with an average relative abundance of $1.46 \times 10^{-1}$ copy ratio. The distribution range was $2.60 \times 10^{-4}-1.02$ copy ratio. The relative abundance range of ARGs in pig manure was $6.54 \times 10^{-5}-5.86 \times 10^{-2}$ copy ratio, and the average relative abundance was $5.44 \times 10^{-3}$ copy ratio. The relative abundance of ARGs in anaerobic digestion liquid was in the range of $1.11 \times 10^{-4}-1.05 \times 10^{-1}$ copy ratio, and the average relative abundance of ARGs was $8.08 \times 10^{-3}$ copy ratio. The relative abundance of ARGs in biogas liquid was slightly higher than that in pig manure. And there were fewer ARGs types in the biogas liquid. An article pointed out that in the anaerobic digestion process, some ARGs may not be degraded, and there is even an enrichment process. So the relative abundance of ARGs in the biogas liquid may be higher than the ARGs in the pig manure [16]. In addition, the occurrence of ARGs was also detected in the soil samples. In the soil samples without anaerobic digestate, the relative abundance range of ARGs was $3.26 \times 10^{-4}-1.47 \times 10^{-1}$ copy ratio, with an average relative abundance of $1.56 \times 10^{-2}$ copy ratio.
But when the soil apply with anaerobic digestate, a total of 20 ARGs were detected, and their relative abundance distribution range was $2.23 \times 10^{-4}-1.22 \times 10^{-1}$ copy ratio, and the average relative abundance increased to $3.47 \times 10^{-}$ 2 copy ratio, indicating that the application of digestate can increase the types and relative abundance of ARGs in the soil [17].

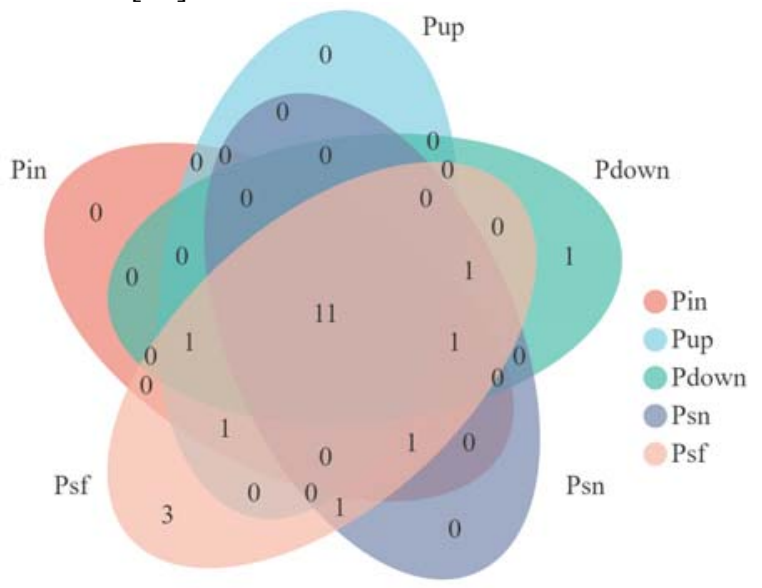

Figure 1. The Venn diagram of ARGs in different samples

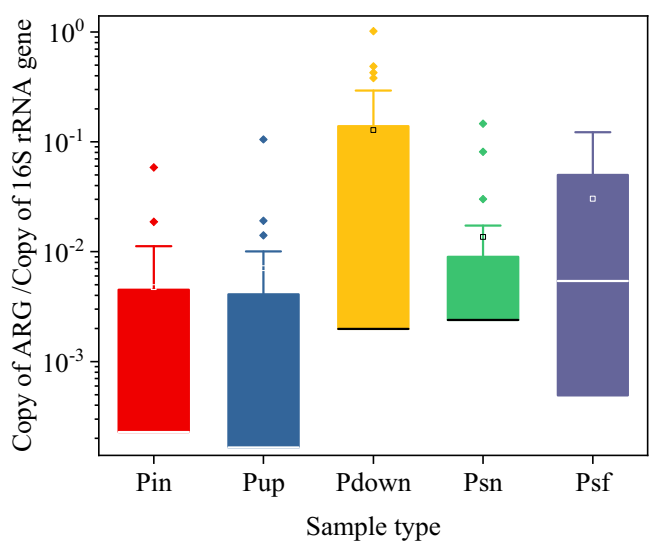

Figure 2. The distribution of total relative abundance of ARGs in different samples

As shown in Figure 3, among all the samples, the relative abundance of aminoglycoside ARG was the highest. The average relative abundance in the 5 samples was as high as $2.35 \times 10^{-1}$ copy ratio, and the relative abundance in biogas residue was as high as 1.02 copy ratio. An article pointed out that the anaerobic digestion process cannot effectively remove the aminoglycoside ARGs, and the aminoglycoside ARGs may have collateral effects with other ARGs under selective pressure [18]. In addition, due to the higher microbial density in the anaerobic digestion residue is beneficial to the spread of ARGs, so aminoglycoside ARGs may be enriched in biogas residue [19]. Other ARGs with high relative abundance were: chloramphenicol, with an average relative abundance of $9.08 \times 10^{-2}$ copy ratio, macrolide-lincosamide-streptogramin (MLS) $7.16 \times 10^{-2}$ copy ratio, multi-drug resistance $1.01 \times 10^{-1}$ copy ratio, sulfonamide $1.10 \times 10^{-1}$ copy ratio, tetracycline $1.24 \times 10^{-1}$ copy ratio, and vancomycin $5.11 \times 10^{-2}$ copy ratio. The 
above types of ARGs are also commonly detected in livestock and poultry manure and their treatment products, so there may be higher occurrences of these ARGs in pig manure, anaerobic digestion products and soils with digestate [7].

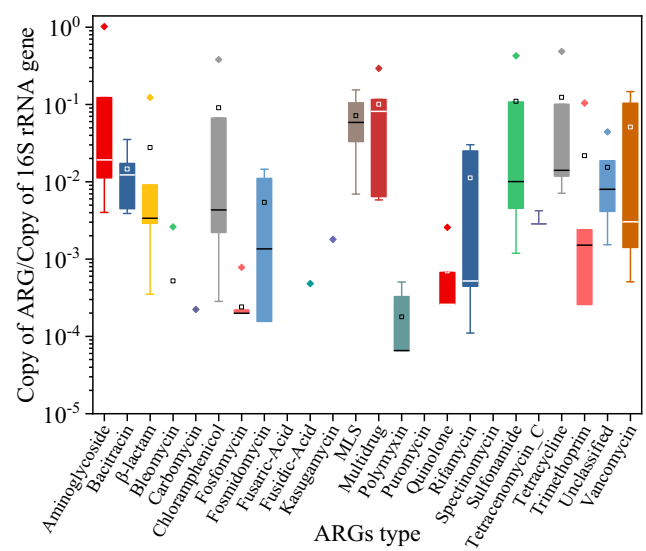

Figure 3. The distribution of relative abundance of different ARGs

\subsection{Change of relative abundance of ARGs in different samples}

It can be seen from Figure 4 that 15 kinds of ARGs were detected in pig manure feed. The relatively abundance of ARGs: aminoglycoside $1.12 \times 10^{-2}$ copy ratio, bacitracin $4.49 \times 10^{-3}$ copy ratio, MLS $5.86 \times 10^{-2}$ copy ratio, multidrug resistant type $6.43 \times 10^{-3}$ copy ratio, sulfonamide $4.53 \times 10^{-3}$ copy ratio, tetracycline $7.14 \times 10^{-3}$ copy ratio, and vancomycin $1.42 \times 10^{-3}$ copy ratio. Comparing the relative abundance of ARGs in pig manure and biogas liquid, the relative abundance of most ARGs increased after anaerobic digestion, and the copy ratio of aminoglycoside increased from $1.12 \times 10^{-2}$ to $1.91 \times 10^{-2}$. The copy ratio of sulfonamide increased from $4.53 \times 10^{-3}$ to $1.01 \times 10^{-2}$, the copy ratio of MLS increased from $5.86 \times 10^{-2}$ to $1.05 \times 10^{-1}$, and the copy ratio of tetracycline increased from $7.14 \times 10^{-3}$ to $1.41 \times 10^{-2}$ copy ratio. However, the relative abundance of some ARGs slightly decreased. For example, the copy ratio of bacitracin decreased from $4.49 \times 10^{-3}$ to $3.89 \times 10^{-3}$, and the copy ratio of multi-drug resistance decreased from $6.43 \times 10^{-3}$ to $5.81 \times 10^{-3}$ copy ratio, vancomycin decreased from $1.42 \times 10^{-3}$ to $5.08 \times 10^{-4}$ copy ratio. However, the relative abundance of overall ARGs increased from $1.14 \times 10^{-1}$ (in pig manure) to $1.70 \times 10^{-1}$ (in biogas liquid). An article pointed out that the anaerobic digestion process has a limited effect on elimination of most ARGs. At the same time, it may have a certain enrichment effect on some ARGs, resulting in the increase of relative abundance of ARGs in biogas liquid [19].

The occurrence content of ARGs in biogas residue was very high. The relative abundance of total ARGs reached 3.07 copy ratio, which was nearly 30 times higher than that in the pig manure and nearly 20 times higher than that in the biogas liquid. ARGs with high relative abundance include: aminoglycoside with a relative abundance of 1.02 copy ratio, which was nearly
100 times higher than that in the pig manure and biogas liquid. The copy ratio of chloramphenicol was $3.80 \times 10^{-1}$, which was 1000 times higher than that in the pig manure, and it was nearly 100 times higher than that in biogas liquid. The copy ratio of sulfonamide was $4.27 \times 10^{-1}$, which was nearly 100 times higher than that in pig manure and 40 times higher than that in biogas liquid. The relative abundance of other ARGs was also nearly 10-1000 times higher than that in pig manure and biogas liquid. It was speculated that most of the microorganisms were deposited in the biogas residue, including the microorganisms carrying ARGs. Thus increased the relative abundance of ARGs in the biogas residue. In addition, the density of microorganisms in the biogas residue was very high, and the microbial gene transfer was more frequent, so ARGs were easier to accumulate in the biogas residue.

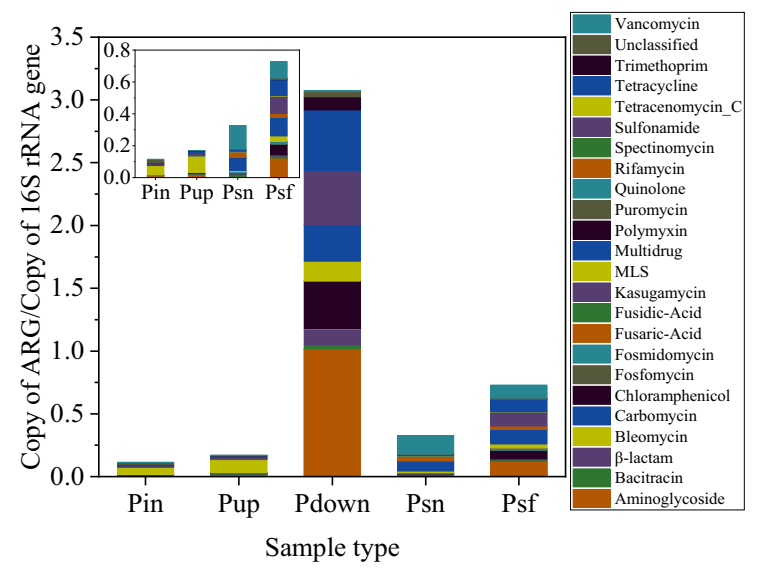

Figure 4. Distribution of ARGs in pig manure, digestate, and soil

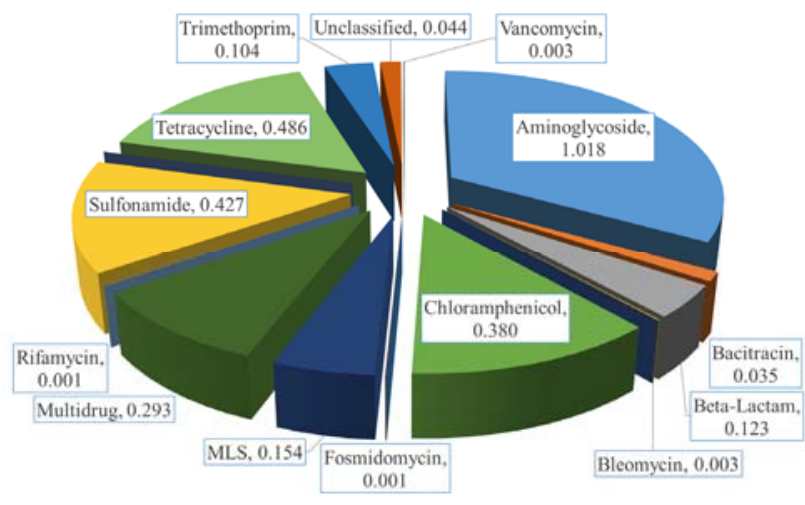

Figure 5. Composition of ARGs in biogas residue

The occurrence of 15 kinds of ARGs were detected in soil samples without anaerobic digestate. The total relative abundance of ARGs was $3.27 \times 10^{-1}$ copy ratio, of which the highest relative abundance was vancomycin, $1.47 \times 10^{-1}$ copy ratio. Other major ARGs were rifamycin $3.01 \times 10^{-2}$ copy ratio, multi-drug resistance $8.14 \times 10^{-2}$ copy ratios, bacitracin $1.72 \times 10^{-2}$ copy ratio, fosmidomycin $1.10 \times 10^{-2}$ copy ratio, and tetracycline $1.18 \times 10^{-2}$ copy ratio. After the application of anaerobic digestate, the relative abundance and types of ARGs had been greatly changed. 20 ARGs were detected in soil 
samples applied with anaerobic digestate. Carbamycin, fosfomycin, fusidic acid, kasugamycin, and trimethoprim ARGs were new compared to the soil without digestate fertilization. Fosfomycin and trimethoprim were exist in the pig manure and anaerobic digestate, so it is presumed that they were brought into the soil from anaerobic digestate of pig manure. The others may be caused by collateral effects of other ARGs and metals or selective pressure. The total relative abundance of ARGs in the soil with digestate fertilization had increased to $7.29 \times 10^{-}$ 2 copy ratio. Among them, aminoglycoside increased from $4.01 \times 10^{-3}$ to $1.22 \times 10^{-1}$ copy ratios. Aminoglycoside was the ARG with the highest relative abundance in soil samples, biogas liquid and biogas residue. This confirmed the hypothesis that digestate have an effect on soil resistance. In addition, the relative abundance of chloramphenicol increased from $2.21 \times 10^{-3}$ to $6.67 \times 10^{-2}$ copy ratio, multi-drug resistance increased from $8.14 \times 10^{-2}$ to $1.16 \times 10^{-1}$ copy ratio, sulfonamide increased from $1.19 \times 10^{-3}$ to $1.08 \times 10^{-1}$ copy ratio, tetracycline increased from $1.18 \times 10^{-2}$ to $1.01 \times 10^{-1}$ copy ratio. Most ARGs increased 3-90 times than that in the soil without digestate application. However, the relative abundance of some ARGs decreased, the copy ratio of vancomycin decreased from $1.47 \times 10^{-1}$ to $1.04 \times 10^{-1}$, the copy ratio of quinolone decreased from $2.57 \times 10^{-3}$ to $6.70 \times 10^{-4}$, the copy ratio of tetracenomycin_C was decreased from $4.22 \times 10^{-3}$ to $2.85 \times 10^{-3}$. The relative abundance of these 3 ARGs in pig manure and digestate were lower than that in soil without digestate application. Thus the soil applying with anaerobic digestate may also dilute the ARGs originally present in the soil $[20,21]$. Studies have pointed out that if the soil fertilizing with amendments that are composted from livestock manure, garden waste and kitchen waste, the resistance of microorganisms will accumulate. So the application of soil amendments come from disposition of livestock manure may increase the antibiotic resistance in the soil [22]. The relative abundance of ARGs in the soil was far less than that in biogas residue, which might because the drug resistance will gradually decrease with the time lapse and hydraulic scouring [21]. However, the problem of ARGs should also be considered when applying anaerobic digestate, especially biogas residue to cropland. And the biogas residue may require further treatment to reduce the occurrence of ARGs.

\section{CONCLUSIONS}

(1) Through sampling and testing, a total of 21 ARGs were detected in pig manure, biogas liquid, biogas residue, and soil samples. 11 of them were shared by all samples, and the most abundant ARG were aminoglycoside. The total relative abundance of aminoglycoside in 5 samples reached 1.18 copy ratio.

(2) The relative abundance of ARGs in biogas residue was much higher than that in other samples. The average relative abundance reached $1.46 \times 10^{-1}$ copy ratio, and the total relative abundance was 3.07 copy ratio. The relative abundance of ARGs was 10-1000 times higher than that in pig manure and biogas liquid. The main types of ARGs were aminoglycoside, chloramphenicol, sulfonamide, tetracycline, and multi-drug resistance.

(3) Anaerobic digestion increased the relative abundance of some ARGs. The relative abundance of total ARGs increased from $1.14 \times 10^{-1}$ to $1.70 \times 10^{-1}$ copy ratio after anaerobic digestion; the application of anaerobic digestate increased some ARGs in the soil. The relative abundance of total ARGs increased from $3.27 \times 10^{-1}$ to $7.29 \times 10^{-1}$ copy ratio.

\section{Acknowledgments}

The work was supported by National water pollution control and control scientific and technological special project (Project No. 2017ZX07103-004)

\section{References}

1. Berendonk $\mathrm{T} \mathrm{U}$, Manaia $\mathrm{C} \mathrm{M}$, Merlin $\mathrm{C}$, et al. Tackling antibiotic resistance: the environmental framework [J]. Nature Reviews: Microbiology, 2015, 13(5):310-7.

2. Luo G, Li B, Li L G, et al. Antibiotic Resistance Genes and Correlations with Microbial Community and Metal Resistance Genes in Full-Scale Biogas Reactors As Revealed by Metagenomic Analysis [J]. Environmental Science \& Technology, 2017, 51(7):4069-4080.

3. Ji X, Shen Q, Liu F, et al. Antibiotic resistance gene abundances associated with antibiotics and heavy metals in animal manures and agricultural soils adjacent to feedlots in Shanghai; China [J]. Journal of Hazardous Materials, 2012, 235-236:178-85.

4. Whitehead T R, Cotta M A Stored swine manure and swine faeces as reservoirs of antibiotic resistance genes [J]. Letters in Applied Microbiology, 2013, 56(4):264-7.

5. Marti R, Scott A, Tien Y C, et al. Impact of manure fertilization on the abundance of antibiotic-resistant bacteria and frequency of detection of antibiotic resistance genes in soil and on vegetables at harvest [J]. Applied and Environmental Microbiology, 2013, 79(18):5701-9.

6. Kanger K, Guilford N G H, Lee H, et al. Antibiotic resistome and microbial community structure during anaerobic co-digestion of food waste, paper and cardboard [J]. FEMS Microbiology Ecology, 2020, 96(2).

7. Wang J, Ben W, Yang M, et al. Dissemination of veterinary antibiotics and corresponding resistance genes from a concentrated swine feedlot along the waste treatment paths $[\mathrm{J}]$. Environment International, 2016, 92-93:317-23.

8. Zhao Q, Liu Y Is anaerobic digestion a reliable barrier for deactivation of pathogens in biosludge? [J]. Science of the Total Environment, 2019, 668:893-902.

9. Tian Z, Zhang Y, Yu B, et al. Changes of resistome, mobilome and potential hosts of antibiotic resistance 
genes during the transformation of anaerobic digestion from mesophilic to thermophilic [J]. Water Research, 2016, 98:261-9.

10. Ma Y, Wilson C A, Novak J T, et al. Effect of various sludge digestion conditions on sulfonamide, macrolide, and tetracycline resistance genes and class I integrons $[\mathrm{J}]$. Environmental Science \& Technology, 2011, 45(18):7855-61.

11. Lu T, Zhang J, Li P, et al. Enhancement of methane production and antibiotic resistance genes reduction by ferrous chloride during anaerobic digestion of swine manure [J]. Bioresource Technology, 2020, 298:122519.

12. Jang H M, Shin J, Choi S, et al. Fate of antibiotic resistance genes in mesophilic and thermophilic anaerobic digestion of chemically enhanced primary treatment (CEPT) sludge [J]. Bioresource Technology, 2017, 244(Pt 1):433-444.

13. Youngquist C P, Mitchell S M, Cogger C G Fate of Antibiotics and Antibiotic Resistance during Digestion and Composting: A Review [J]. Journal of Environment Quality, 2016, 45(2):537-45.

14. Zhang J, Lu T, Wang Z, et al. Effects of magnetite on anaerobic digestion of swine manure: Attention to methane production and fate of antibiotic resistance genes [J]. Bioresource Technology, 2019, 291:121847.

15. Sui Q, Zhang J, Chen M, et al. Distribution of antibiotic resistance genes (ARGs) in anaerobic digestion and land application of swine wastewater [J]. Environmental Pollution, 2016, 213:751-759.

16. Gurmessa B, Pedretti E F, Cocco S, et al. Manure anaerobic digestion effects and the role of pre- and post-treatments on veterinary antibiotics and antibiotic resistance genes removal efficiency [J]. Science of the Total Environment, 2020, 721:137532.

17. $\mathrm{Pu} \mathrm{C}$, Liu $\mathrm{H}$, Ding $\mathrm{G}$, et al. Impact of direct application of biogas slurry and residue in fields: In situ analysis of antibiotic resistance genes from pig manure to fields [J]. Journal of Hazardous Materials, 2018, 344:441-449.

18. Zhao R, Feng J, Liu J, et al. Deciphering of microbial community and antibiotic resistance genes in activated sludge reactors under high selective pressure of different antibiotics [J]. Water Research, 2019, 151:388-402.

19. Ju F, Li B, Ma L, et al. Antibiotic resistance genes and human bacterial pathogens: Co-occurrence, removal, and enrichment in municipal sewage sludge digesters [J]. Water Research, 2016, 91:1-10.

20. Duan M, Gu J, Wang X, et al. Factors that affect the occurrence and distribution of antibiotic resistance genes in soils from livestock and poultry farms [J]. Ecotoxicology and Environment Safety, 2019, 180:114-122.

21. Lin H, Chapman S J, Freitag T E, et al. Fate of tetracycline and sulfonamide resistance genes in a grassland soil amended with different organic fertilizers [J]. Ecotoxicology and Environment Safety, 2019, 170:39-46.

22. Scott A, Tien Y C, Drury C F, et al. Enrichment of antibiotic resistance genes in soil receiving composts derived from swine manure, yard wastes, or food wastes, and evidence for multiyear persistence of swine Clostridium spp [J]. Canadian Journal of Microbiology, 2018, 64(3):201-208. 